\title{
MAXIPOL: A Balloon-borne Experiment for Measuring the Polarization Anisotropy of the Cosmic Microwave Background Radiation
}

\author{
B. R. Johnson ${ }^{1}$, M. E. Abroe ${ }^{1}$, P. Ade ${ }^{3}$, J. Bock ${ }^{4}$, J. Borrill' ${ }^{7,9}$, \\ J. S. Collins ${ }^{2}$, P. Ferreira ${ }^{5}$, S. Hanany ${ }^{1}$, A. H. Jaffe ${ }^{8}$, \\ T. Jones ${ }^{1}$, A. T. Lee ${ }^{2,6}$, L. Levinson ${ }^{10}$, T. Matsumura ${ }^{1}$, B. Rabii ${ }^{2}$, T. Renbarger ${ }^{1}$,

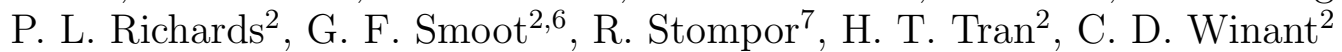 \\ ${ }^{1}$ School of Physics and Astronomy, University of Minnesota, Minneapolis, MN, USA \\ ${ }^{2}$ Department of Physics, University of California, Berkeley, CA, USA \\ ${ }^{3}$ Department of Physics and Astronomy, University of Wales, Cardiff, UK \\ ${ }^{4}$ Jet Propulsion Laboratory, Pasadena, CA, USA \\ ${ }^{5}$ Astrophysics 83 Theoretical Physics, University of Oxford, Oxford, UK \\ ${ }^{6}$ Physics Division, Lawrence Berkeley National Lab, Berkeley, CA, USA \\ ${ }^{7}$ Computational Research Division, Lawrence Berkeley National Lab, Berkeley, CA, USA \\ ${ }^{8}$ Astrophysics Group, Blackett Lab, Imperial College, London, UK \\ ${ }^{9}$ Space Sciences Laboratory, University of California, Berkeley, CA, USA \\ ${ }^{10}$ Department of Particle Physics, Weizmann Institute of Science, Rehovot, Israel
}

October 30, 2018

\begin{abstract}
We discuss MAXIPOL, a bolometric, balloon-borne experiment designed to measure the E-mode polarization anisotropy of the cosmic microwave background radiation (CMB) on angular scales of $10^{\prime}$ to $2^{\circ}$. MAXIPOL is the first CMB experiment to collect data with a polarimeter that utilizes a rotating half-wave plate and fixed wire-grid polarizer. We present the instrument design, elaborate on the polarimeter strategy and show the instrument performance during flight with some time domain data. Our primary data set was collected during a 26 hour turnaround flight that was launched from the National Scientific Ballooning Facility in Ft. Sumner, New Mexico in May 2003. During this flight five regions of the sky were mapped. Data analysis is in progress.
\end{abstract}

\section{Introduction}

MAXIPOL is a bolometric, balloon-borne experiment designed to measure the E-mode polarization anisotropy in the cosmic microwave background radiation (CMB). The MAXIPOL instrument is a reimplementation of the hardware from the successful CMB temperature anisotropy experiment MAXIMA $[1,2,3]$. While the MAXIMA telescope and data electronics remained largely unchanged, the receiver was converted into a polarimeter by retrofitting it with a rotating half-wave plate (HWP) and a fixed wire-grid polarizer.

MAXIPOL has flown twice from NASA's National Scientific Ballooning Facility in Ft. Sumner, New Mexico. The first flight, MAXIPOL-0, launched in September 2002 and the second, MAXIPOL-1, in May 2003. In this paper we discuss the science goals of the experiment, the hardware implementation (Section 2), the flights (Section 3), the noise and potential systematic errors (Section 4).

The goal of MAXIPOL is to measure the peaks in the E-mode (EE) and temperature-E-mode (TE) cross correlation power spectra between $\ell=300$ and $\ell=1000$. To accomplish this goal, MAXIPOL mapped the $I, Q$ and $U$ Stokes parameters of $2^{\circ}$ wide "bow tie" shaped regions of the sky with $10^{\prime}$ resolution. Detection of the polarization anisotropy of the CMB was recently reported by DASI [4] and WMAP [5].

\section{Instrument Description}

Many subsystems in the MAXIPOL instrument have already been thoroughly detailed in previous MAXIMA publications $[1,6,7,8]$. This discussion will focus primarily on the new MAXIPOL-specific hardware elements that were retrofitted into the MAXIMA instrument. 


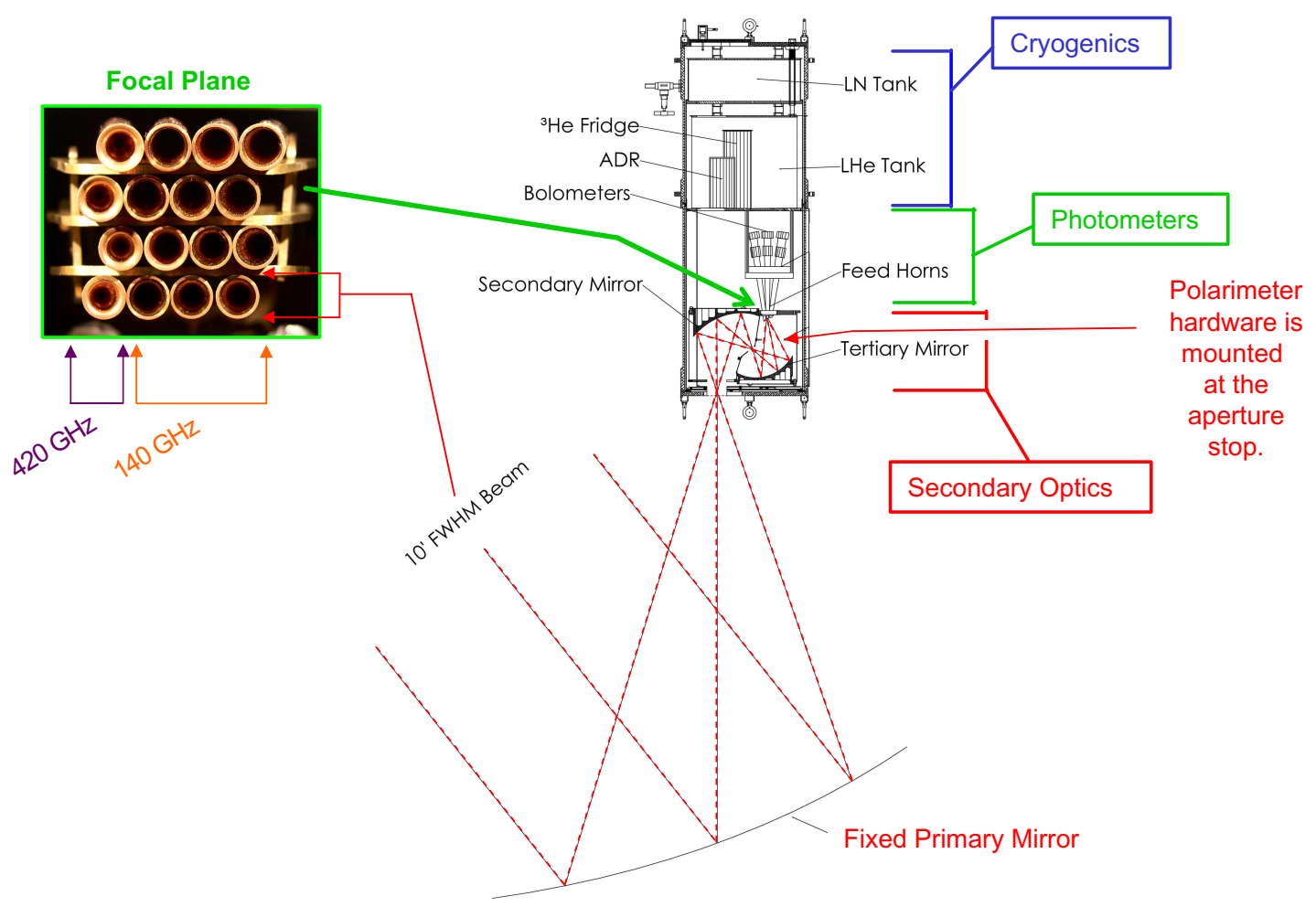

Figure 1: A cross-sectional view of the MAXIPOL receiver (Section 2).

\section{$2.1 \quad$ Overview}

MAXIPOL employs a three mirror, f/1 Gregorian telescope with a $1.3 \mathrm{~m}$ off-axis parabolic primary mirror. The elliptical secondary and tertiary reimaging mirrors (21 and $18 \mathrm{~cm}$ in diameter, respectively) are held at liquid helium temperatures inside the receiver to reduce radiative loading on the bolometers. To keep the instrumental polarization properties of the telescope constant, all telescope mirrors are fixed with respect to each other for all observations. ${ }^{1}$

Light from the sky is reimaged to a $4 \times 4$ array of horns at the focal plane. Observations are made in bands centered on $140 \mathrm{GHz}$ and $420 \mathrm{GHz}(\Delta \nu \simeq 30 \mathrm{GHz})$. The twelve $140 \mathrm{GHz}$ photometers are optimized to measure the $\mathrm{CMB}$ and the four $420 \mathrm{GHz}$ photometers are used to monitor foreground dust contamination. The 10' FWHM Gaussian beam shape for the $140 \mathrm{GHz}$ photometers is defined by a smooth walled, single-mode conical horn and a cold Lyot stop; the $420 \mathrm{GHz}$ photometers employ multi-mode Winston horns. The bolometers are maintained at $100 \mathrm{mK}$ by an adiabatic demagnetization refrigerator [10] and a $300 \mathrm{mK}{ }^{3}$ He refrigerator. A photograph of the focal plane and a cross-sectional view of the inside of the cryostat is shown in Figure 1. For reference, the HWP and wire-grid polarizer that will be discussed in Section 2.2 are mounted near the Lyot stop and at the focal plane, respectively.

The telescope was focused before flight by mapping the detector beams with a chopped, 100 Watt halogen filament imaged at infinity by a 38 inch on-axis parabolic mirror. An absorptive, 0.75 inch thick plug of Eccosorb MF110 was inserted in the optical path at the intermediate focus of the telescope to attenuate the intensity of warm loads during lab measurements. This attenuator was anti-reflection (AR) coated with a 0.015 inch thick sheet of etched Teflon. The calculated transmission was $\sim 1 \%$ at $140 \mathrm{GHz}[9]$.

A schematic of the MAXIPOL instrument can be seen in Figure 2. This illustration shows the payload without sun shielding so the telescope, receiver and attitude control subsystems are visible. Before flight, sun shielding was installed to protect all subsystems from solar radiation during daytime observations and to shield the telescope and receiver from spurious signals caused by sunlight and RF transmitters. The baffling was made of Celotex aluminized foam sheeting and was painted white on all sun and earth-facing surfaces. We selected a white paint pigmented with $\mathrm{TiO}_{2}$ because this material has low solar absorptivity $(\sim 10 \%)$ and high infrared emissivity $(\sim 90 \%)$ - a combination that compensated for the loss of convective cooling in the low-pressure balloon environment by providing adequate radiative cooling. This sun shield design successfully maintained all instrument subsystems within nominal temperature specifications during the daytime portion of the flight. A large aluminum ground shield (also not illustrated) was mounted to the inner frame to shield the main beam of the telescope from terrestrial emission.

Telescope attitude was feedback controlled. The flywheel mounted at the top of the gondola moved the telescope in

\footnotetext{
${ }^{1}$ The primary mirror was chopped in azimuth during MAXIMA observations.
} 


\section{MAXIPOL Instrument}

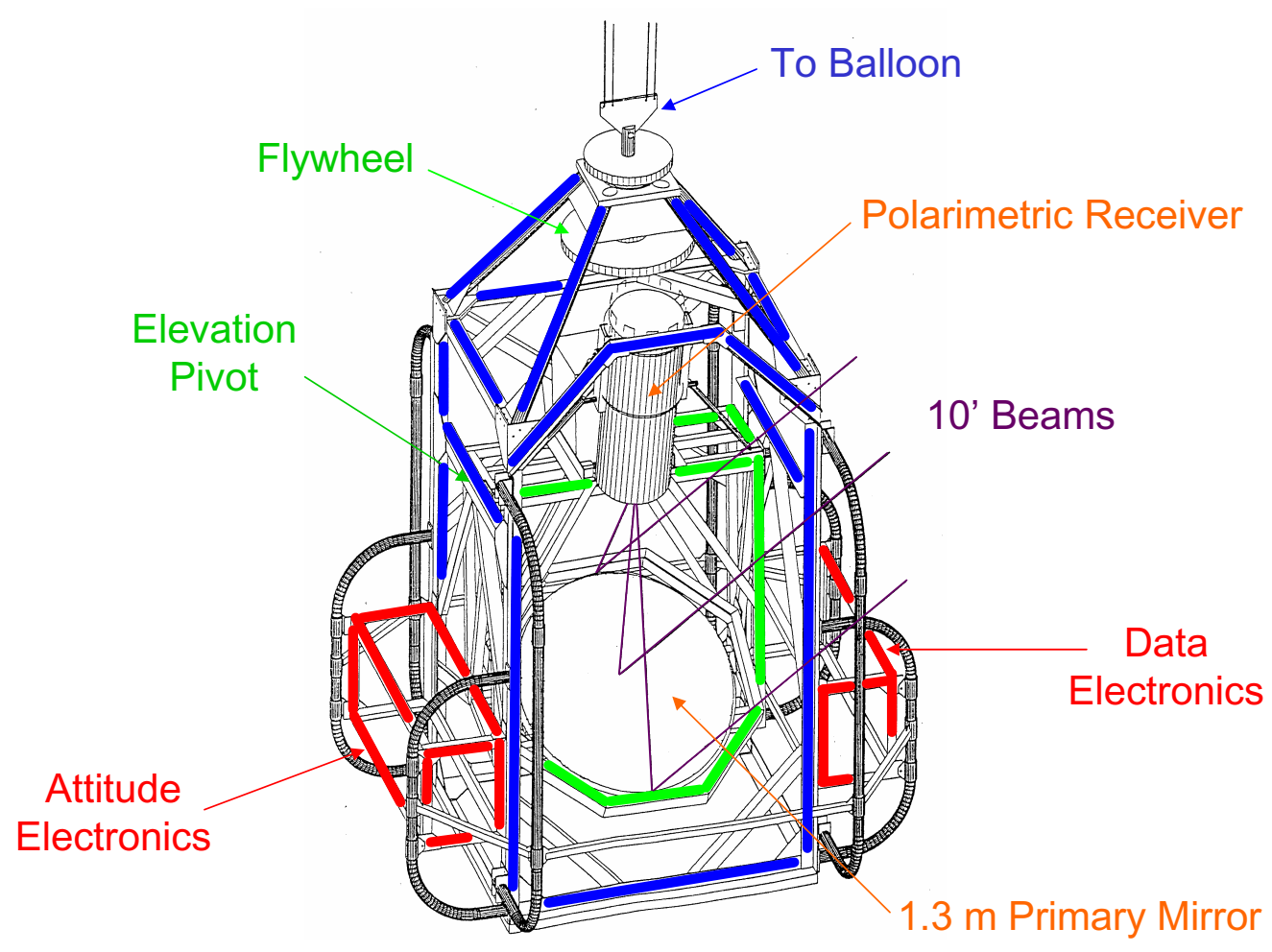

Figure 2: The MAXIPOL instrument without baffling (Section 2).

azimuth while the elevation angle was adjusted with a linear actuator arm that nodded the entire inner frame. A second motor mounted at the very top of the gondola further assisted in moving the telescope in azimuth by torquing the payload against the balloon cabling. The azimuth feedback-loop relied on gyros and a magnetometer and the elevation feedback relied on a 16-bit optical encoder. The magnetometer was calibrated before flight; the offset was measured to within a degree and the non-linearity was mapped and stored in a lookup table that was used by the on-board pointing computer during flight for making fine corrections. A second lookup table was also implemented to account for variations in the magnetic field of the Earth as a function of longitude and latitude.

Pointing reconstruction for data analysis relies on the position of a reference star in one of two boresight Cohu 4910 CCD cameras. The camera used during daytime observations was filtered with a $695 \mathrm{~nm}$ Schott glass filter and fitted with a $500 \mathrm{~mm}$ Promaster Spectrum 7 reflective lens that provided a $0.72^{\circ}$ by $0.55^{\circ}$ field-of-view; the unfiltered nighttime camera used a $50 \mathrm{~mm}$ Fujinon lens that provided a $7.17^{\circ}$ by $5.50^{\circ}$ field-of-view. Pixel size for the daytime and nighttime cameras was $0.084^{\prime}$ by $0.069^{\prime}$ and $0.84^{\prime}$ by $0.69^{\prime}$, respectively. The small field-of-view and the filter on the daytime camera were necessary to improve the ratio of star to sky brightness. With the combination, we detected stars of apparent visual magnitude 2 at balloon altitude. The two cameras and the telescope were aligned before flight to within a quarter of a degree.

Bolometer data and housekeeping signals were multiplexed into a single data stream that was telemetered to fixed ground stations during flight. These signals were monitored in real time to ensure nominal operation of the instrument and because the cryogenic system needed to be manually cycled.

A new on-board data recorder was added to the experiment for the MAXIPOL-1 flight after a NASA data transmitter failed during MAXIPOL-0. The data recorder, which was custom designed and built by the Weizmann Institute of Science in Israel, consists of NIM modules each containing an Altera FPGA chip and up to 128 Intel 16 MB flash memory chips. With an uncompressed serial data rate of $160 \mathrm{kbps}$ each module is capable of storing 28.4 hours of data. Individual modules can be daisy-chained to each other to increase the total recording capacity. The FPGA chip reads the incoming data stream and when it detects a pre-programmed frame structure it controls the storage of the data on the memory chips. It also controls the post-flight export of the data from the memory chips into a computer through a standard parallel port. Power consumption during steady-state data recording is less than 0.25 Watt (at $5 \mathrm{~V}$ ), and modules that are idle require only about 0.05 Watt. For MAXIPOL-1 two modules containing 96 memory chips provided a total recording capacity of 42.6 hours. Approximately 28 hours of pre-flight, ascent and at-float data were recorded. 


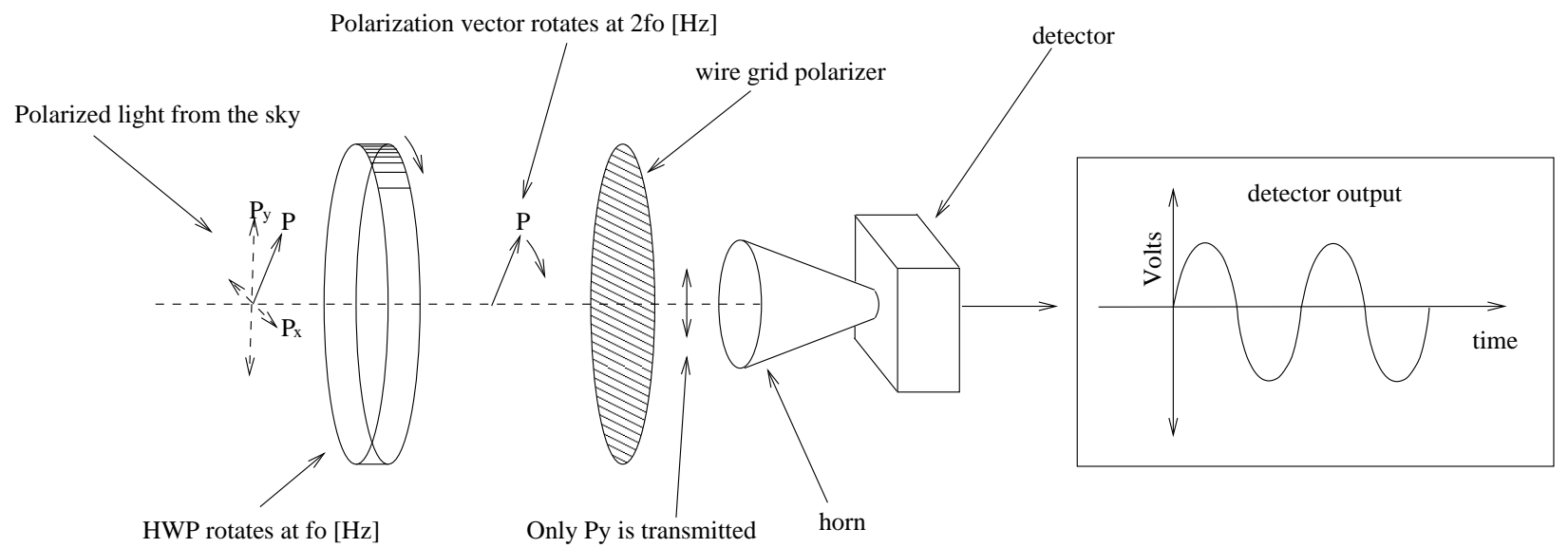

Figure 3: The polarimeter strategy employed by MAXIPOL (Section 2.2).

\subsection{Polarimeter Strategy}

MAXIPOL analyzed the polarization of the millimeter-wave sky with a rotating HWP and fixed wire-grid polarizer. While this technique is a well-known standard in astronomy, it is the first implementation in a CMB experiment. The strategy is illustrated in Figure 3.

Monochromatic linearly polarized light that passes through a HWP rotating at a frequency $f_{0}$ emerges linearly polarized with its orientation rotating at $2 f_{0}$. If this light then propagates through a fixed polarizer and its intensity is subsequently measured, the resulting data stream will exhibit sinusoidal modulation at $4 f_{0}$. The amplitude of this modulation depends on the level of polarization of the incident radiation. Perfectly polarized light will maximize the amplitude and perfectly unpolarized light will yield no modulation.

The advantage of HWP polarimetry is that each detector in the array makes an independent measurement of the Stokes parameters of the incoming radiation. In addition, this technique rejects systematic errors. Spatial polarization variations on the sky translate to temporal amplitude variations in the $4 f_{0}$ signal because the telescope is scanning. Therefore, the polarization anisotropy data will reside in the sidebands of the $4 f_{0}$ signal in Fourier space. Any spurious or systematic signals appearing in the data stream outside of this $4 f_{0}$ frequency band can be filtered away with software during data analysis (see Section 4).

The HWP is inherently a monochromatic device so the behavior described above applies only to the frequencies $\nu=m c / 2 t \Delta n$ where $\Delta n$ is the difference between the ordinary and extraordinary index of refraction in the birefringent crystal, $t$ is the propagation length through the crystal, $m$ is an odd integer and $c$ is the speed of light. Linearly polarized light at other frequencies emerges from the crystal elliptically polarized. We calculated the HWP thickness that would minimize the fraction of elliptically polarized intensity and thereby optimize the overall polarimeter efficiency. To do this, we found the maximum of the product of the expected efficiencies for the 140 and $420 \mathrm{GHz}$ photometers as a function of crystal thickness. These expected efficiency curves incorporated the spectral breadth of the photometers and the convergence of rays as they propagate through the HWP. The HWP design that resulted from this calculation is discussed in Section 2.3.

To ascertain the polarimeter efficiency, a polarized load was analyzed in the lab before flight. For this measurement, a wire-grid polarizer was mounted on the cryostat window with its transmission axis oriented parallel to that of the focal plane polarizer. Thermal radiation from a $273 \mathrm{~K}$ ice bath was chopped at $\sim 6.5 \mathrm{~Hz}$ with a $300 \mathrm{~K}$ aluminum chopper blade covered with 0.25 inch thick Eccosorb LS-14 foam. The HWP was then discretely stepped by hand in $\sim 5^{\circ}$ intervals. Twenty seconds of data were collected at each HWP orientation. The amplitude of the chopped signal in the bolometer time stream for one typical photometer was measured with a software lock-in analysis and then plotted in Figure 4. A nine parameter model consisting of sine waves for the first four harmonics of $f_{0}$ was then fit to the data (solid curve); fit parameters included the amplitudes and phases of each sine wave and an overall offset. The level of polarization was then calculated from the fit parameters using the standard definition $P=\left(A_{\max }-A_{\min }\right) /\left(A_{\max }+A_{\min }\right)$ where $A$ is the amplitude of the $4 f_{o}$ signal. This calibrated load was measured to be $86 \%$ polarized. This corresponds to an overall polarimeter efficiency of $89 \%$ which is in agreement with predictions that take into account the HWP thickness, the known spectral response of the $140 \mathrm{GHz}$ photometers, the convergence of rays at the aperature stop of the telescope and the wire-grid polarizer efficiency.

Incident unpolarized light can become partially polarized inside the instrument if it reflects off of the telescope mirrors at oblique angles. In addition, emission from the mirrors may also be partially polarized. To assess the level of instrumental polarization the procedure outlined above was repeated with unpolarized light. We found the instrumental polarization to be $1 \%$ for a typical $140 \mathrm{GHz}$ channel. 


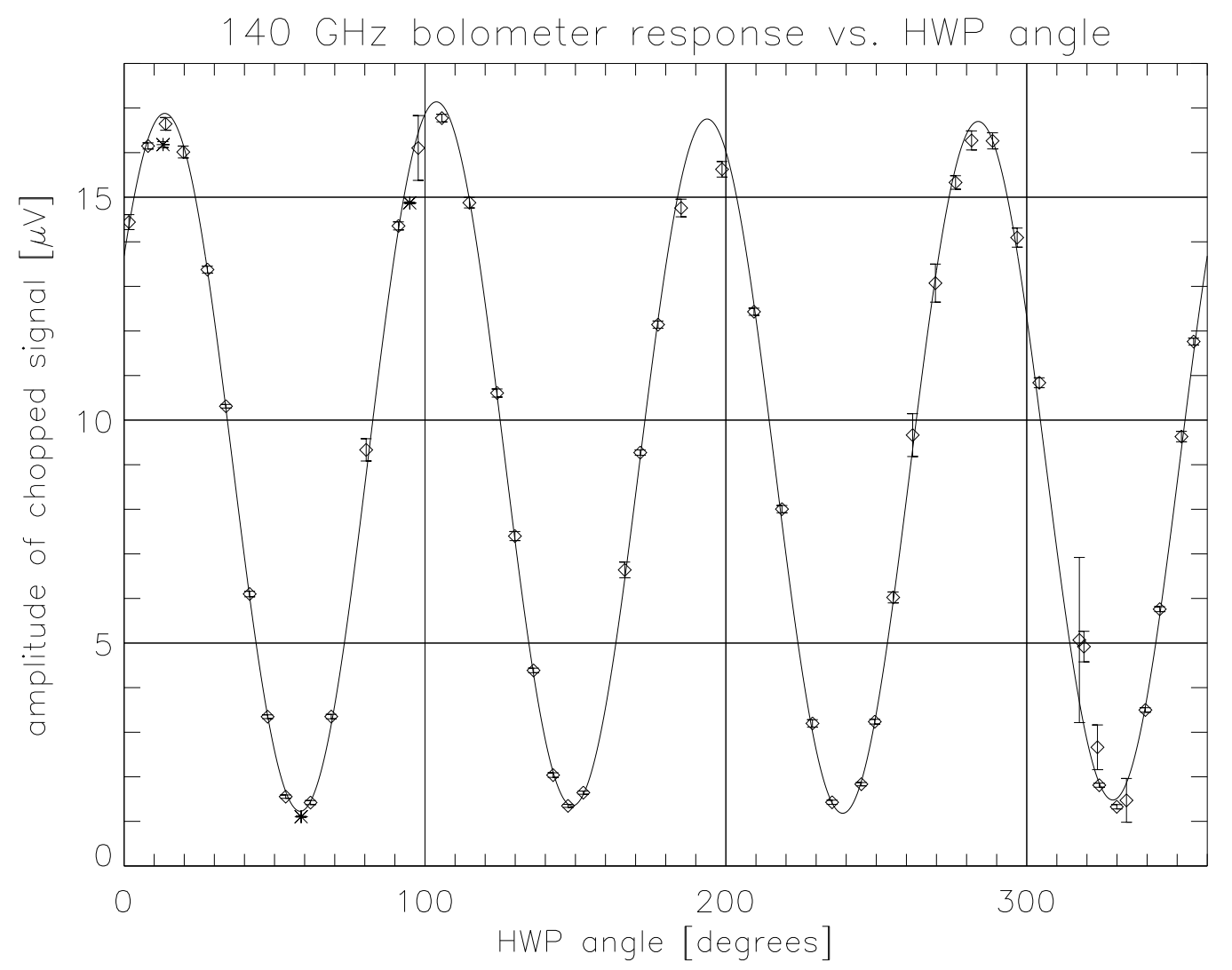

Figure 4: A lab measurement of a polarized load. The setup for this measurement is described in Section 2.2. The solid curve plotted is the best fit model comprised of $f_{0}, 2 f_{0}, 3 f_{0}$ and $4 f_{0}$ sine waves; the nine free parameters used in the fit include the amplitude and phase of each sine wave and an overall offset. For this model, the reduced $\chi^{2}=1.22$ for 42 degrees of freedom. From the fit parameters, we calculated the polarimeter efficiency to be $89 \%$.

\subsection{Half-Wave Plate and Wire-Grid Polarizer}

The $3.4 \mathrm{~mm}$ thick A-cut sapphire HWP was AR coated with a 0.013 inch thick wafer of Herasil to maximize transmission. The Herasil was bonded to the sapphire with Eccobond 24, an unfilled, low viscosity epoxy that was used to achieve glue layers as thin as 0.0005 inches. For MAXIPOL-0 we AR coated the HWP with a 0.010 inch thick layer of Stycast $2850 F T$. The switch from Stycast to Herasil was made because Herasil thermally contracts in a way that is more compatible with sapphire.

Since the AR coating was not birefringent, the two incident polarization orientations had different coefficients of reflection; this differential reflection gave rise to a rotation synchronous signal at a frequency of $2 f_{0}$. To minimize this effect, we calculated the AR coating thickness that would minimize the difference in reflection coefficients given the spectral breadth of the $140 \mathrm{GHz}$ photometers, the thickness of the eccobond 24 layer and the oblique incidence of rays. Because the $2 f_{0}$ signal resides out of the polarization signal bandwith around $4 f_{0}$ it is not a source of systematic error (see Section 4 ).

The focal plane wire-grid polarizer, made by Buckbee-Mears, was constructed from electroformed 0.0002 inch diameter gold wires bonded to 0.0015 inch thick Mylar film at 250 lines per inch. This flexible material was mounted to a rigid "roof-shaped" frame that was positioned over the horn openings. This "roof-shaped" polarizer reflected the unwanted polarization orientation out of the optical path and into blocks of millimeter-wave absorbing material [11] mounted on either side of the focal plane. This design reduced spurious signals due to reflections.

\subsection{HWP Drivetrain}

The HWP rotated at $\sim 2 \mathrm{~Hz}$ during both MAXIPOL flights. This speed was selected because it provided eight measurements of the of Stokes parameters for one beam resolution element per scan period while avoiding any significant $4 f_{0}$ signal attenuation from the $\sim 10 \mathrm{~ms}$ bolometer time constant. During operation, this rotation speed proved to be vibrationally gentle; it did not excite any detectable microphonic signals in the bolometer data.

The HWP was center turned near the Lyot stop of the telescope by a 0.078 inch diameter driveshaft (see Figure 5). This driveshaft penetrated the tertiary mirror and the cryostat shell and was turned through a low-temperature ferrofluid rotary vacuum feedthrough (Ferrofluidics FE51-122190A) by a feedback controlled Kollmorgen U9M4 Servo Disc DC motor with high-altitude brushes mounted outside the receiver. The orientation of the motor shaft, and therefore the HWP, was 


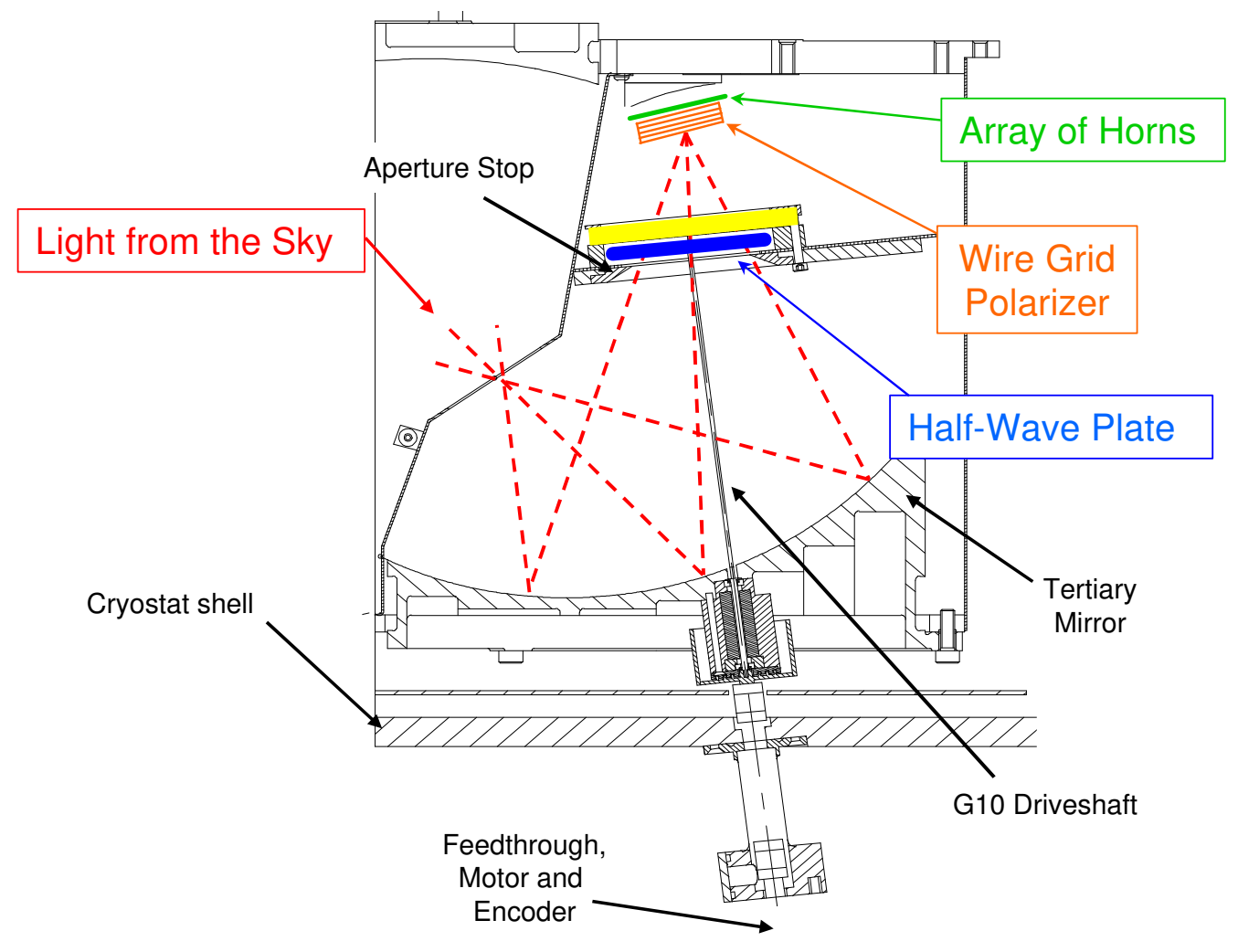

Figure 5: A cross-sectional view of the MAXIPOL polarimeter (Section 2.4).

measured with a 16-bit Gurley A25S optical encoder.

The HWP was held in place near the Lyot stop with a Rulon-J sleeve bearing embedded in a 0.4 inch thick disk of Zote foam. A sapphire bearing was used in MAXIPOL-0 but was later found to exhibit less favorable vibrational properties. This foam disk was mounted in the optical path perpendicular to the chief ray. A polished, hardened-steel sewing needle was passed through a center drilled hole in the HWP; the assembly resembled a toy top. One end of the needle was slipped into the Rulon-J sleeve and the other end was rigidly coupled to the driveshaft with 907 epoxy.

The driveshaft had three main parts. Between the HWP and the tertiary mirror, the driveshaft was made of thin wall G10 tubing (wall thickness $\simeq 0.005 \mathrm{in}$ ). The thin fiberglass G10 material minimized both the thermal load on the HWP and the optical cross-section of the exposed driveshaft, while retaining the desired torsional driveshaft stiffness. A bearing assembly was mounted at the back of the tertiary mirror to act as a thermal intercept and to provided necessary mechanical stability for the driveshaft. The bearing was made of Rulon-J (Vespel SP3) for MAXIPOL-1 (MAXIPOL-0). Inside this bearing, the driveshaft was made of polished steel, sputter coated with $\mathrm{MoS}_{2}$; the shaft material was cromoly steel (titanium nitride coated tungsten carbide) for MAXIPOL-1 (MAXIPOL-0). Between the tertiary mirror and the rotary vacuum feedthrough at the cryostat shell the driveshaft was again made of G10 tubing to minimize the thermal load on the HWP and the liquid helium bath.

Laboratory testing was carried out to test the vibrational properties of the drivetrain assembly at liquid helium temperatures. A mock-up of the drivetrain was constructed and installed in a liquid helium cryostat. With this setup, two bearing materials, Rulon-J and Vespel SP3, were studied with microphones mounted near the bearings inside the cryostat. Rulon-J was chosen as the flight drivetrain bearing material because of the low noise performance it exhibited over several consecutive days of testing.

\section{MAXIPOL Flights}

\subsection{MAXIPOL-0}

During the $\sim 22$ hour MAXIPOL-0 flight the NASA data transmitter failed sporadically because of a broken solder connection. As a result, only a few $\sim 10$ minute sections of bolometer data were successfully recorded; we did not realize enough integration time for CMB measurements. The flight did provide us with the opportunity to check the in-flight polarimeter 


\begin{tabular}{ccccc} 
& $\begin{array}{c}\text { Observation Length } \\
\text { in [hours] }\end{array}$ & $\begin{array}{c}\text { Average Dust } \\
\text { Level }[\mu K]\end{array}$ & $\begin{array}{c}\text { RMS Dust } \\
\text { Level }[\mu K]\end{array}$ & Observation Time \\
\hline Beta Ursae Minoris & 7.5 & 20 & 3.2 & night \\
Polaris & 2.25 & 173 & 23 & day \\
Gamma Urase Majoris & 2.5 & 11 & 2.5 & day \\
Gamma Virgo & 0.5 & 22 & 3.2 & day \\
Arcturus & 2.0 & 27 & 5.1 & day \\
\hline
\end{tabular}

Table 1: MAXIPOL-1 scan regions. Five bow tie shaped regions of the sky were mapped during MAXIPOL-1 by tracking five different guide stars. The observation length and the expected dust contribution for each region [12] is presented.

performance and to test the new daytime pointing camera, the sun shielding strategy and the HWP driveshaft motor and encoder.

\subsection{MAXIPOL-1}

During the MAXIPOL-1 flight, we executed four different types of telescope scans: a planet scan, a dipole scan, a CMB scan and a foreground dust scan.

During the planet scan, the gondola yawed sinusoidally $2.5^{\circ}$ peak-to-peak in azimuth at a slowly-rising elevation for $\sim 1$ hour. The scan period was 18 seconds. During this time, Jupiter passed through the field-of-view of the instrument and was detected by the bolometers. This data set will be used to map the beam shape of each photometer and calibrate the bolometer time streams given the known millimeter-wave intensity of Jupiter. We performed both daytime and nighttime observations of Jupiter.

The CMB dipole was scanned by rotating the gondola $360^{\circ}$ in azimuth while holding the telescope at a constant elevation of $36^{\circ}$ for 22 minutes. A second $\sim 10$ minute scan was performed at an elevation of $50^{\circ}$. The period of a single rotation was 18 seconds. With this data set we will calibrate the bolometer time streams given the large, known CMB dipole signal.

For the CMB and dust scans, the telescope tracked a guide star as it swept across the sky. Simultaneously, the gondola yawed $2^{\circ}$ peak-to-peak in azimuth with a period of 10 seconds. This telescope motion combined with the inherent sky rotation produced bow tie shaped maps. To improve cross-linking, the telescope elevation dithered periodically about the elevation of the guide star by $\pm 0.2^{\circ}$ with an elevation change ocurring every 10 minutes. Table 1 summarizes the scan length and the expected dust contribution for the five regions observed during MAXIPOL-1. Regions with strong dust contamination will be used to characterize this foreground signal.

To monitor the bolometer temperature dependence of the calibration, a fixed intensity millimeter-wave lamp mounted near the focal plane was switched on for 10 seconds every 22 minutes. The relationship between the magnitude of the subsequent bolometer response and the known bolometer temperature will be ascertained during data analysis. The responsivity of each bolometer sample will be interpolated given this relative calibration and the absolute Jupiter and CMB dipole calibrations.

\section{Flight Performance}

The purpose of this section is to demonstrate the viability of the instrument. Five minutes of MAXIPOL-0 time stream data from one $140 \mathrm{GHz}$ photometer are plotted in the upper left panel of Figure 6. When this data is replotted versus HWP angle, the systematic offsets become apparent (upper right panel).

Plotted in the lower left panel is the power spectrum of this data set. The first peak appears at $f_{0}$ and the subsequent peaks appear at the harmonics of $f_{0}$. Laboratory measurements show that the source of the $f_{0}$ signal is predominantly thermal emission from the G10 drivetrain. This thermal emission signal contributes at all harmonics in the plot though it is subdominant in the $2 f_{0}$ and $4 f_{0}$ peaks. The dominant $2 f_{0}$ signal comes from the differential reflection effect discussed in Section 2.3; the dominant $4 f_{0}$ signal comes from the instrumental polarization signal discussed in Section 2.2 .

As discussed in Section 2.2, polarization anisotropy measurements will appear in the sidebands of the $4 f_{0}$ signal. If the amplitude of the systematic offset at $4 f_{0}$ varies, this modulation may mimic polarization signals from the sky. To measure its stability, we lock-in on the $4 f_{0}$ offset with software using a reference created from the HWP optical encoder data stream. The time dependence of the locked-in data provides a measure of the stability of the amplitude of the $4 f_{0}$ offset. The power spectrum of this locked-in data is plotted in the lower right panel of Figure 6 . In the bandwidth of interest spanning 0 to $1 \mathrm{~Hz}$ we recovered the nominal instrument noise level of $\sim 10 \mathrm{nV} \mathrm{rms}_{\mathrm{s}} / \sqrt{\mathrm{Hz}}$; this bandwith was set by the scan speed of the telescope, the beam size and the angular size of the expected structure on the sky. We therefore conclude that variations in the known systematic offsets will contribute less than the detector noise to the the MAXIPOL data. 

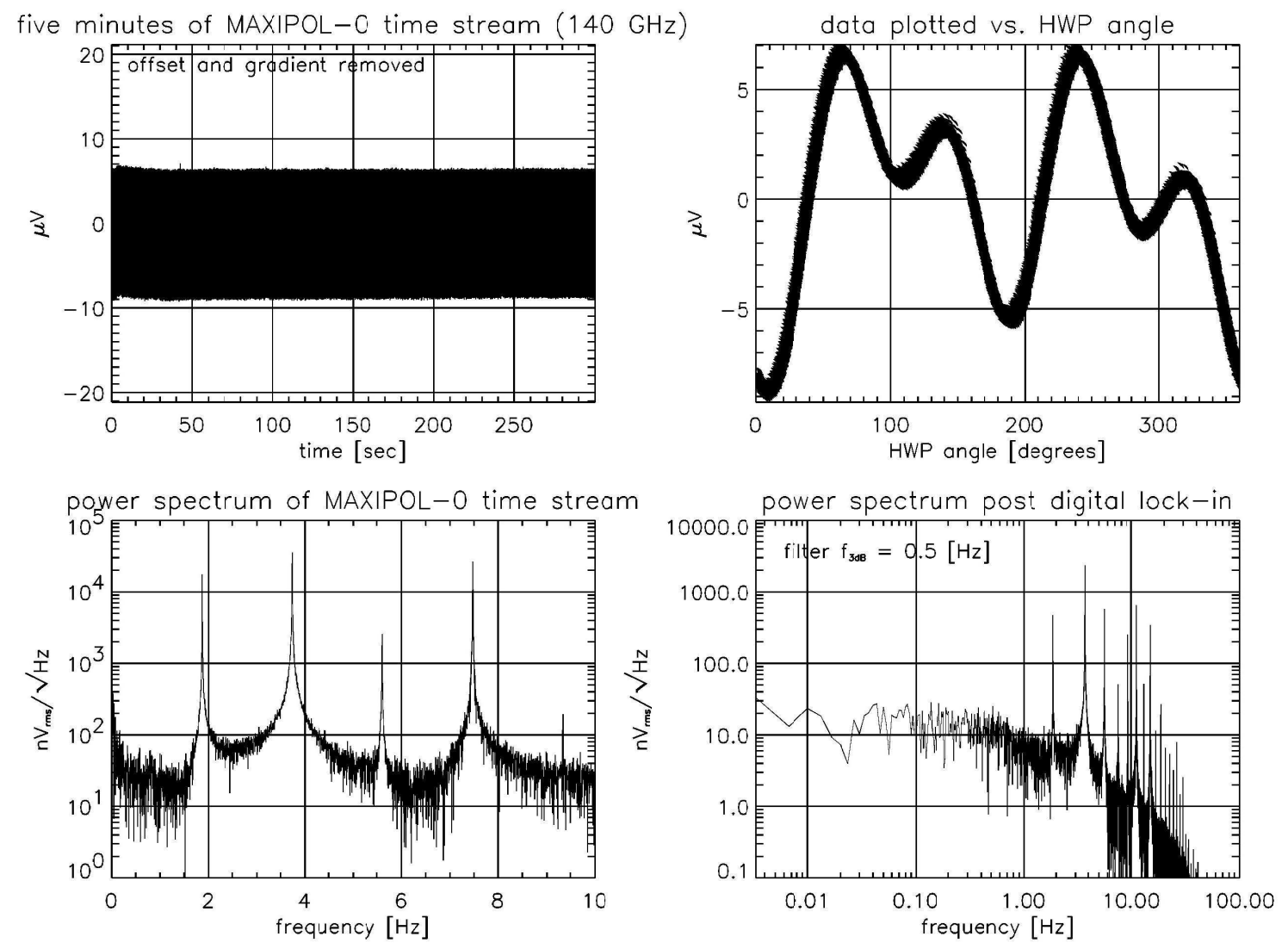

Figure 6: A software lock-in analysis of five minutes of MAXIPOL-0 data (See the discussion in Section 4).

\section{Acknowledgements}

We thank Danny Ball and the other staff members at NASA's National Scientific Ballooning Facility in Ft. Sumner, New Mexico for their outstanding support of the MAXIPOL program. We would also like to thank the members of the Electronics \& Data Acquisition Unit in the Faculty of Physics at the Weizmann Institute of Science in Rehovot, Israel. MAXIPOL is supported by NASA Grants NAG5-12718, NAG5-3941 and S-92548-F; a NASA GSRP Fellowship for B. Johnson; the Land McKnight Professorship at the University of Minnesota, Twin Cities for S. Hanany; and the Miller Institute at the University of California, Berkeley for H. Tran.

\section{References}

[1] Hanany, S., et al. 2000. ApJ, 545:L5-L9

[2] Lee, A. T., et al. 2001. ApJ, 561:L1-L5

[3] Stompor, R., et al. 2001. ApJ, 561:L7-L10

[4] Kovac, J.M., et al. 2002. Nature, 420, 772

[5] Kogut, A., et al. 2003. ApJ, accepted. astro-ph/0302213.

[6] Lee, A. T., et al. 1998. The Proceedings of the "3 K Cosmology" Conference, ed. F. Melchiorri. astro-ph/9903249.

[7] Winant, C. D. 2003. Ph.D. Thesis. University of California, Berkeley.

[8] Rabii, B. 2002. Ph.D. Thesis. University of California, Berkeley.

[9] Peterson, J. B. \& Richards, P. L. 1984. Int. J. Infrared Millimeterwaves, vol. 5, no. 12, pp. 1507-1515.

[10] Hagmann C. \& Richards, P.L. 1995. Cryogenics, vol. 35, no. 5, pp. 303-309.

[11] Bock, J. J. 1994. Ph.D. Thesis. University of California, Berkeley.

[12] Jaffe, A. H., et al. 1999. Invited review in "Microwave Foregrounds", eds. A. de Oliveira-Costa \& M. Tegmark. ASP, San Francisco. astro-ph/9903248. 